\title{
Behavior of quantum entropies in polaronic systems
}

\author{
C.A. Perroni, V. Marigliano Ramaglia, and V. Cataudella \\ CNR-SPIN and Dipartimento di Scienze Fisiche, \\ Università degli Studi di Napoli "Federico II", \\ Complesso Universitario Monte Sant'Angelo, \\ Via Cintia, I-80126 Napoli, Italy
}

\begin{abstract}
Quantum entropies and state distances are analyzed in polaronic systems with short range (Holstein model) and long range (Fröhlich model) electron-phonon coupling. These quantities are extracted by a variational wave function which describes very accurately polaron systems with arbitrary size in all the relevant parameter regimes. With the use of quantum information tools, the crossover region from weak to strong coupling regime can be characterized with high precision. Then, the linear entropy is found to be very sensitive to the range of the electron-phonon coupling and the adiabatic ratio. Finally, the entanglement entropy is studied as a function of the system size pointing out that it not bounded, but scales as the logarithm of the size either for weak electronphonon coupling or for short range interaction. This behavior is ascribed to the peculiar coupling induced by the single electron itinerant dynamics on the phonon subsystem.
\end{abstract}

\section{INTRODUCTION}

In the last years quantum entanglement has attracted enormous interest as a key physical resource at the basis of quantum information processing. [1] In particular, attention has been devoted to clarify and quantify quantum entanglement in many-body systems since entanglement measures provide insights on the quantum correlations of many-body functions. 2] For example, entanglement entropy and its scaling properties are currently used in order to better characterize quantum phase transitions. In the case of local couplings between degrees of freedom, the entropy of the reduced state of a subregion grows like the boundary area of the subregion, and not like its volume, that is known as area law. 3. However, close to a transition, this law is violated since the entropy becomes divergent as a function of the system size. The size scaling very often follows a logarithm law.

Tools given by quantum information have been especially important for mesoscopic systems where several schemes have been proposed for detection and measurement of entanglement. [4] Very recently, a microwavefrequency mechanical oscillator has been cooled to its ground state with high probability and coupled to a quantum bit. [5, 6] This coupling preserves the quantum states and allows a time-domain control of the system. In these experiments the maximum number of phonons in the relevant mechanical mode is very low, so that one expects that the coupling between mesoscopic resonator and quantum bit is not strong. Moreover, a scenario has been proposed in order to detect entanglement of a mechanical resonator and a qubit in a nanoelectromechanic setup. [7]

The realization of such devices where many quantum bits or multiple electronic states are coupled to mesoscopic or macroscopic mechanical objects is far from being easy. Therefore, it is important to make theoretical studies of such systems elucidating the role of the coupling between electrons and oscillators and their entanglement properties. In particular, the dimension of the system becomes a very important parameter for the analysis. Instead of considering artificial devices, one can analyze compounds already existing in nature where a array of microscopic oscillators is present in mesoscopic or macroscopic systems. Therefore, the aim of this work is to study from a quantum information perspective a system relevant in many areas of condensed and nanoscopic matter: the polaron, i.e. a single electron (with many accessible states in a bulk crystal or a quantum dot) interacting with lattice phonons. [8] It has been proved that it does not show any self-trapping phase transition with increasing electron-phonon $(e l-p h)$ coupling, but a crossover between solutions with small extended (large polaron) and large localized (small polaron) lattice deformations. [9] Up to now, only quantum entropies have been analyzed for polaronic systems with small size and short range $(S R) e l-p h$ interactions. 10, 11] In addition to quantum entropies, in this work, distance measures are studied in polaronic models with arbitrary size taking the free electron as reference state. We will use a variational approach that is very accurate in all the coupling and adiabaticity regimes. 12, 13] By means of these tools, the precise position of the crossover region between weak and strong coupling regime is identified.

Polaron studies extend the analysis of simpler spinboson models. 14 The entanglement for polaron is very interesting also for other reasons: it can be considered as a measure of decoherence of the electronic state due to the coupling with phonons or dephasing of the phononic state induced by the interaction with the single electron. From this point of view, studies of polaronic entanglement are relevant in mesoscopic systems such as quantum dots in polar semiconductors that have been proposed as systems for quantum processing. 15] Moreover, in these systems, the $e l-p h$ interaction is not local. Therefore, in this paper, we have for the first time analyzed the effect 
of long range $(L R) e l-p h$ couplings on entanglement amount. It is found that quantum entropies are strongly dependent on the adiabatic ratio and range of the interaction. The final part of the paper will focus on the size scaling of the entanglement entropy. Even if the system is not critical, the entropy of polaronic systems is unbounded, and it scales as the logarithm of the size either for weak $e l-p h$ coupling or for local interactions.

\section{MODELS AND VARIATIONAL APPROACH}

The prototype model with $S R$ local coupling is the well known Holstein one, 16] while that with $L R$ interactions is the Fröhlich one. 17] In particular a discrete version of the Fröhlic model will be studied. [18] The Hamiltonian of the polaronic systems is

$$
H=-t \sum_{<i, j>} c_{i}^{\dagger} c_{j}+\omega_{0} \sum_{i} a_{i}^{\dagger} a_{i}+H_{e l-p h} .
$$

In Eq. (11) $c_{i}^{\dagger}\left(c_{i}\right)$ denotes the electron creation (annihilation) operator at site $i$, whose position vector is indicated by $\vec{R}_{i}$, and the symbol $<>$ denotes nearest neighbors linked through the transfer integral $t$. The operator $a_{i}^{\dagger}$ $\left(a_{i}\right)$ represents the creation (annihilation) operator for phonon on the site $i$, and $\omega_{0}$ is the frequency of the optical local phonon modes. Both the $S R$ and $L R$ models can be described by the general $e l-p h$ Hamiltonian $H_{e l-p h}$

$$
H_{e l-p h}=\alpha \omega_{0} \sum_{i, j} f\left(\left|\vec{R}_{i}-\vec{R}_{j}\right|\right) c_{i}^{\dagger} c_{i}\left(a_{j}+a_{j}^{\dagger}\right),
$$

where $\alpha$ controls the strength of $e l-p h$ coupling, and $f\left(\left|\vec{R}_{i}-\vec{R}_{j}\right|\right)$ is the interacting force between an electron on the site $i$ and an ion displacement on the site $j$. The units are such that the lattice parameter $a=1$ and $\hbar=1$.

The Hamiltonian (1) reduces to the Holstein model for

$$
f\left(\left|\vec{R}_{i}-\vec{R}_{j}\right|\right)=\delta_{\vec{R}_{i}, \vec{R}_{j}},
$$

while in the $L R$ case [18] the interaction force is given by

$$
f\left(\left|\vec{R}_{i}-\vec{R}_{j}\right|\right)=\left(\left|\vec{R}_{i}-\vec{R}_{j}\right|^{2}+1\right)^{-\frac{3}{2}} .
$$

Through the matrix element $M_{\vec{q}}$, defined as the lattice Fourier transform of $\alpha \omega_{0} f\left(\left|\vec{R}_{i}\right|\right)$, one defines the polaronic shift $E_{p}=\sum_{\vec{q}} M_{\vec{q}}^{2} / \omega_{0}$ and the coupling constant $\lambda=E_{p} / z t$, with $z$ lattice coordination number, that represents a natural measure of the strength of the $e l-p h$ coupling for any range of the interaction. Another important parameter of polaronic systems is the adiabatic ratio $\gamma=\omega_{0} / t$.

We adopt a variational approach previously proposed [12, 13] for the study of systems with variable range $e l-p h$ interactions and arbitrary size. Not only ground state energies, but also effective masses and spectral weights calculated with this approach have been compared with the results of numerical approaches finding excellent agreement. The trial wave functions are translational invariant Bloch states obtained by taking a superposition of localized states centered on different lattice sites

$$
\left|\psi_{\vec{k}}^{(i)}>=\frac{1}{\sqrt{N}} \sum_{\vec{R}_{n}} e^{i \vec{k} \cdot \vec{R}_{n}}\right| \psi_{\vec{k}}^{(i)}\left(\vec{R}_{n}\right)>
$$

where

$$
\left|\psi_{\vec{k}}^{(i)}\left(\vec{R}_{n}\right)>=e^{S^{(i)}\left(\vec{R}_{n}\right)} \sum_{m} \phi_{\vec{k}}^{(i)}\left(\vec{R}_{m}\right) c_{m+n}^{\dagger}\right| 0>,
$$

with

$$
S^{(i)}\left(\vec{R}_{n}\right)=\sum_{\vec{q}}\left[h_{\vec{q}}^{(i)}(\vec{k}) a_{\vec{q}} e^{i \vec{q} \cdot \vec{R}_{n}}+h . c .\right] .
$$

In the last equations, $N$ is the number of lattice sites (corresponding to the dimensionality of the electron Hilbert space), the apex $i=w, s$ indicates the weak and strong coupling polaron wave function, respectively, $\mid 0>$ denotes the electron and phonon vacuum state, $h_{\vec{q}}^{(i)}(\vec{k})$ are the phonon distribution functions and $\phi_{\vec{k}}^{(i)}\left(\vec{R}_{m}\right)$ are variational parameters defining the spatial broadening of the electronic wave function. For each function, the variational minimization becomes accurate extending the electron wave function up to a few neighbors.

The ground state properties are determined by considering as trial state $\left|\psi_{\vec{k}}\right\rangle$ a linear superposition of the weak and strong coupling wave functions

$$
\mid \psi_{\vec{k}}>=\frac{A_{\vec{k}}\left|\bar{\psi}_{\vec{k}}^{(w)}>+B_{\vec{k}}\right| \bar{\psi}_{\vec{k}}^{(s)}>}{\sqrt{A_{\vec{k}}^{2}+B_{\vec{k}}^{2}+2 A_{\vec{k}} B_{\vec{k}} S_{\vec{k}}}}
$$

where $\mid \bar{\psi}_{\vec{k}}^{(i)}>$ is the normalized wave function and $S_{\vec{k}}=<$ $\bar{\psi}_{\vec{k}}^{(w)} \mid \bar{\psi}_{\vec{k}}^{(s)}>$ is the overlap factor of the two wave functions. In Eq.(8) $A_{\vec{k}}$ and $B_{\vec{k}}$ are two additional variational parameters which provide the relative weight of the weak and strong coupling solutions for any particular value of $\vec{k}$. In the rest of this work, we will study the onedimensional ground state corresponding to $\vec{k}=k=0$ in the physically relevant adiabatic regime $\gamma<1$.

\section{RESULTS}

The main quantity extracted from the wave function is the phonon-traced electron density operator $\rho_{e l}$

$$
\rho_{e l}=\operatorname{Tr}_{p h}\left[\left|\psi_{k=0}><\psi_{k=0}\right|\right]
$$


where $\operatorname{Tr}_{p h}$ denotes the trace over the phonon degrees of freedom. In order to analyze the entanglement between electron and phonon, one can use the linear entropy $S_{L}$

$$
S_{L}=1-\operatorname{Tr}_{e l}\left[\left(\rho_{e l}\right)^{2}\right],
$$

where $T r_{e l}$ stands for the trace over the electronic degrees of freedom. [19] In Fig. 1, we report the linear entropy as a function of the coupling $\lambda$ for $S R$ and $L R$ interactions. It is zero for a free electron, then it increases with $\lambda$ reaching the saturation value $1-1 / N$ that marks the transition to the totally mixed state. For the Holstein model, $S_{L}$ reaches the saturation value at a value of $\lambda$ slightly larger than unity. In the $L R$ case, $S_{L}$ increases due to the larger entanglement between electron and phonons. Moreover, the crossover between the weak and strong coupling regimes is smoother. [13] All these features make the linear entropy a very important quantity to measure the change of polaron features as function of the coupling $\lambda$.

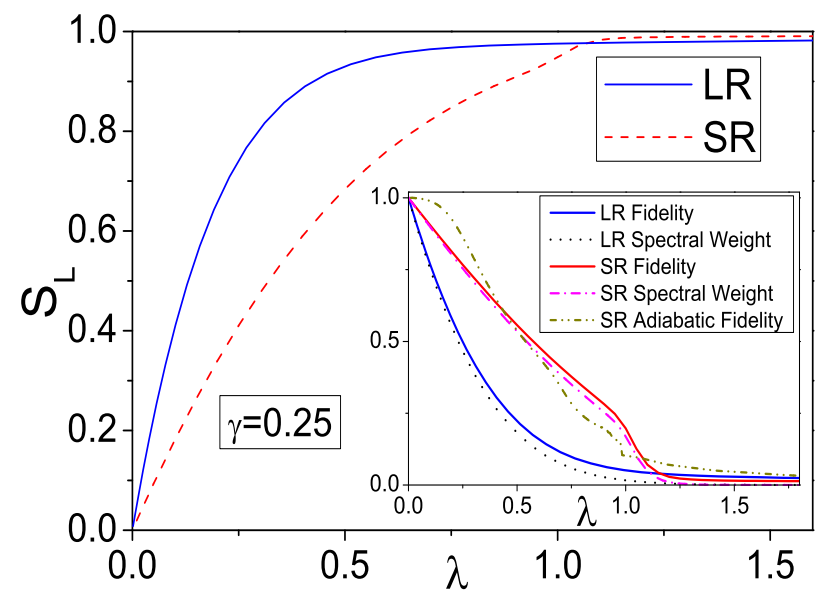

FIG. 1. Linear entropy as a function of $\lambda$ for $S R$ and $L R$ ranges of interaction at $\gamma=0.25$ and $N=128$. In the inset, fidelity and spectral weight as a function of $\lambda$. The fidelity in the fully adiabatic regime is also shown.

Another important quantity for the analysis of polaronic systems is the fidelity $F$

$$
F=\operatorname{Tr}_{e l}\left[\left(\rho_{\text {free-el }}\right)\left(\rho_{e l}\right)\right],
$$

where $\rho_{\text {free-el }}$ is the free electron density operator appropriate for periodic boundary conditions. [20] The fidelity considered here is a measure of distance between the polaron and the free electron state. In the inset of Fig.1, we show the fidelity for $L R$ and $S R$ case. It is 1 for $\lambda=0$ and decreases with increasing $\lambda$ (for $S R$ it is linear in the intermediate regime) up to a value close to zero in the strong coupling regime. Therefore, in the maximally entangled state, single electron and phonons are so strongly coupled that free electron features have completely disappeared. In the $L R$ case, due to the larger mixing between degrees of freedom, the fidelity shows a marked tendency towards strong coupling features.

In the inset of Fig.1, the fidelity is compared to the ground state spectral weight $Z=\mid\left\langle\psi_{k=0}\left|c_{k}^{\dagger}=0\right| 0\right\rangle$ $\left.\right|^{2}$ ), with $c_{k}^{\dagger}$ electron creation operator in the momentum representation. $Z$ measures how much the quasi-particle is different from the free electron $(Z=1)$. A small value of it indicates a strong mixing of electronic and phononic degrees of freedom. $Z$ and $F$ (in the same inset) share the same behavior as function of $\lambda$ for both $S R$ and $L R$ case.

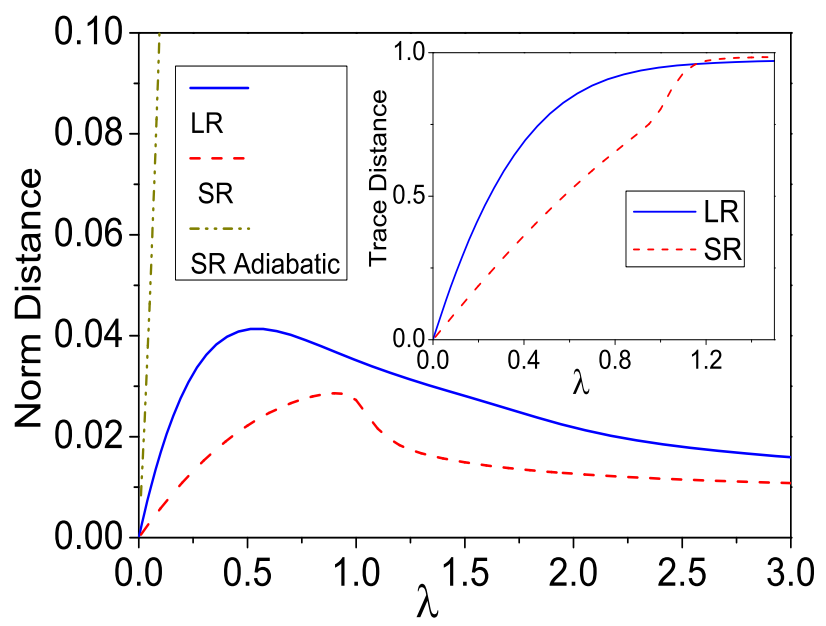

FIG. 2. Norm distance as function of $\lambda$ for $S R$ and $L R e l-p h$ coupling at $\gamma=0.25$ and $N=128$. The same quantity in the fully adiabatic regime is also shown. In the inset, the trace distance as function $\lambda$ for the same values of parameters.

The spectral weight has been used to distinguish qualitatively a crossover regime $(0.1<Z<0.9)$ between the quasi-free-electron one $(0.9<Z<1)$ and strong coupling one $(Z<0.1)$. [12, 13] Our analysis shows that a new quantum measure, the norm distance, is able to characterize in a quantitative way the crossover region. The norm distance is defined as the eigenvalue norm of the difference density operator $\sigma=\rho_{e l}-\rho_{e l-f r e e}$ : $\|\sigma\|_{\eta}=\max _{i}\left|\eta_{i}\right|$, with $\eta_{i}$ eigenvalues of $\sigma$. [19] In Fig. 2 , the norm distance is reported for $S R$ and $L R$ couplings. In the first case, it is peaked at a value of $\lambda$ slightly smaller than unity. The $L R$ case it is much more interesting since the distance shows a maximum at about $\lambda=0.5$, which corresponds to an intermediate value of the fidelity and spectral weight. Finally, it is possible to evaluate the trace distance as the trace norm of $\sigma / 2$. 19] This function shows a behavior very similar to the linear entropy. From the comparison of the two distances, it emerges that the maximum eigenvector of $\sigma$ is always a fraction of the sum of all the others. Therefore, the 
peak shown in the norm distance is to be ascribed to the peculiar structure of the wave-function in this regime.

It is important to access the difference of the results between adiabatic regime and fully adiabatic limit. In the limit $\omega_{0} \rightarrow 0$, the phonon fields are classical and the only relevant $e l-p h$ coupling is $\lambda$. Clearly, quantum entropies vanish. However, it is still possible to study the fidelity $F$ (shown in the inset of Fig.1). $F$ in this regime shows an abrupt change at $\lambda=1$ for the $S R$ case. This corresponds to the self-trapping transition towards a very localized state which breaks the translation invariance. In Fig. 2, we report the norm distance in the full adiabatic limit for the $S R$ case. It is strongly larger than its corresponding quantity with quantum phonons. Actually, in strong coupling, one eigenvalue is close to unity, that relative to the electron localized on a single site.

Since quantum entropies are zero in the fully adiabatic regime, we analyze the behavior of the linear entropy close to this limit. In Fig. 3, we report the linear entropy for the $S R$ and $L R$ case as a function of the adiabatic ratio $\gamma$ for two values of the $e l-p h$ constant $\alpha$. The entropies get larger with increasing the adiabatic ratio. They are strongly dependent on $\alpha$ and on the range of the $e l-p h$ interaction. Indeed, for large values of $\alpha$, the parameter $\lambda$ increases very fast as a function of $\gamma$. Therefore, the linear entropy reaches the saturation value close to unity. Actually quantum entropies are very sensitive to quantum phonon fluctuations, so that they could be used as analyzer of the quantum nature of the oscillators.

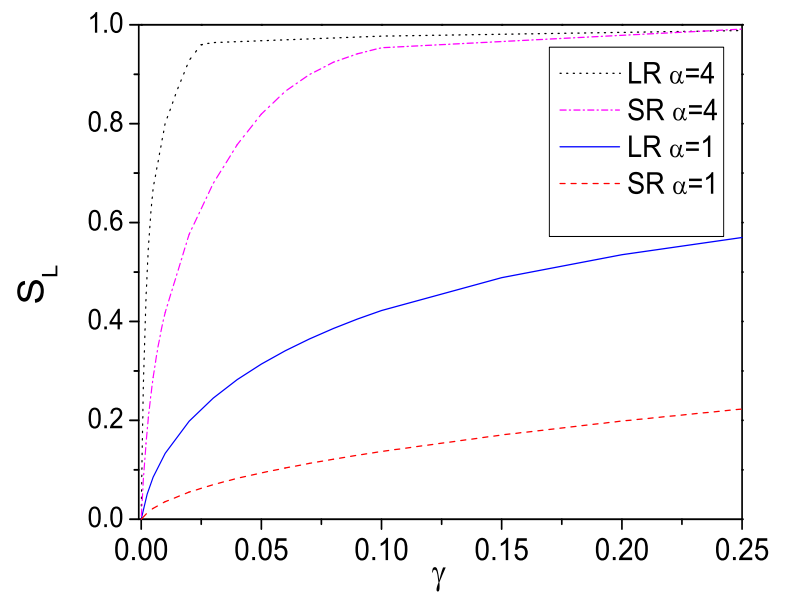

FIG. 3. Linear entropy as a function of the adiabatic ratio $\gamma$ for several values of the $e l-p h$ coupling constant $\alpha$ at $N=128$.

In the last part of the paper, we will analyze the entanglement or von Neumann entropy $S_{V N}$

$$
S_{V N}=\operatorname{Tr}_{e l}\left[\left(\rho_{e l}\right) \ln \left(\rho_{e l}\right)\right]=S_{V N}^{e l}=S_{V N}^{p h o n}
$$

in particular its dependence on the size $N$. The equivalence between electron and phonon entropy is due to the fact that the starting point is a bipartite pure state. 19] $S_{V N}$ is zero for decoupled electron and phonon degrees of freedom, and has the maximum value of $\ln (N)$, since $N$ is the dimension of the smallest subsystem, the electron one.

At fixed size, the linear and von Neumann entropy are monotonic functions of each other. Therefore, $S_{V N}$ increases as a function of $\lambda$ up to a saturation value in the strong coupling regime when the state is maximally entangled. We have found that, in this limit, for both $S R$ and $L R$ interactions, $S_{V N}$ reaches the maximum value, so that it is not bounded but scales as $\ln (N)$. This behavior in the strong coupling regime is due, in our opinion, to the peculiar "long-range" coupling induced by the single electron itinerant dynamics on the phonon subsystem.

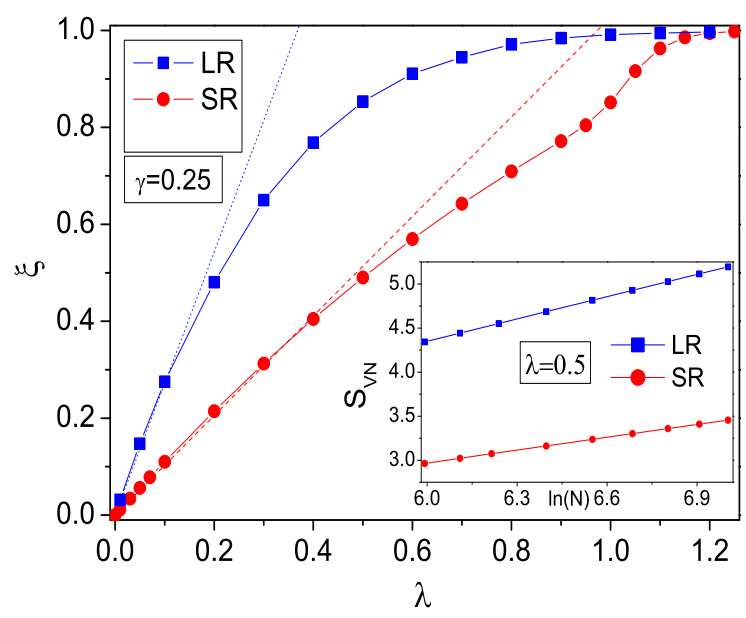

FIG. 4. The quantity $\xi$ as function of $\lambda$ at $\gamma=0.25$. In the inset the von Neumann entropy as a function of the system size $N$ at fixed value of $\lambda$.

This result is even more surprising since it is not limited to the strong coupling limit. The scaling proportional to $\ln (N)$ is obtained also in the weak and intermediate regime. At the second order of the perturbation theory in the $e l-p h$ coupling, in the limit of large $N$, $S_{V N} \rightarrow \bar{N}_{p h} \ln (N)$, where $\bar{N}_{p h}$ is the perturbative average number of excited virtual phonons (linear as function of $\lambda$ ). We have found by means of an accurate fitting procedure that, in all the regimes, $S_{V N}$ scales as $\xi \ln (N)+a$, where $\xi$ (see Fig. 4) and $a$ depend on the $e l-p h$ coupling. In the inset of Fig. 4, we report the von Neumann entropies at fixed value of $\lambda$ as function of the size $N$. With increasing the $e l-p h$ coupling, $\xi$ deviates from the linear dependence, and in the crossover regime it curves towards the value of unity for strong coupling. Indeed, $\xi$ behaves as the linear entropy shown in Fig.1. 
One of the main results of this work is that the entanglement entropy is unbounded and scales as a logarithm of the size in all the regimes. This result is valid also for $L R$ el $-p h$ interaction. Actually, $S_{V N}$ cannot increase more than $\ln (N)$ due to the dimensional constraint of the electron Hilbert space. This study allows to quantify the amount of the entanglement of realistic systems, for example quantum dots in polar semiconductors, where $L R$ polaronic effects can be important. In these systems, the entanglement can be also related to the measure of decoherence of the electronic state induced by the interactions with phonons. As a result of this study, one can estimate that the entanglement entropy due to $e l-p h$ coupling scales as the logarithm of size with a proportionality constant that varies in a simple way as a function of the parameters.

The experimental detection of entanglement is difficult for the bulk, but feasible for nanostructures. Recently, an electronic measurement has been suggested in order to detect entanglement between a qubit and an oscillator making use of an atomic point contact. [7] Furthermore, again in the case of the interaction between qubit and resonator, time domain control has been used in order to controllably create a phonon in the resonator and to observe the exchange of this excitation between qubit and oscillator. [6] Effects of electric fields have been also analyzed for polarons in bulk semiconductors. 21] The pulse induces not only coherent lattice vibrations but also velocity drift oscillations of the electron. This is again a demonstration of the more complex entanglement behavior between electron and phonon degrees of freedom that has been the focus of this work.

We acknowledge R. Fazio for a critical reading of part of the manuscript.

[1] R. Horodecki, P. Horodecki, M. Horodecki and K. Horodecki, Rev. Mod. Phys. 81, 865 (2009).
[2] L. Amico, R. Fazio, A. Osterloh, and V. Vedral, Rev. Mod. Phys. 80, 517 (2008).

[3] J. Eisert, M. Cramer and M. B. Plenio, Rev. Mod. Phys. 82, 277 (2010).

[4] C.W.J. Beenakker, in Proceedings of the International School of Physics "E. Fermi" edited by G. Casati, D. L. Shepelyansky, and P. Zoller (IOS, Amsterdam, 2006), Vol. 162.

[5] T. Rocheleau, T. Ndukum, C. Macklin, J. B. Hertzberg, A. A. Clerk, and K. C. Schwab, Nature 463, 72 (2010).

[6] A. D. OConnell, M. Hofheinz, M. Ansmann, Radoslaw C. Bialczak, M. Lenander, Erik Lucero, M. Neeley, D. Sank, H. Wang, M. Weides, J. Wenner, John M. Martinis, and A. N. Cleland, Nature 464, 697 (2010).

[7] T.L. Schmidt, K. Brkje, C. Bruder, and B.Trauzettel, Phys. Rev. Lett. 104, 177205 (2010).

[8] Proceedings of the International School of Physics "E. Fermi", Course CLXI edited by G. Iadonisi, J. Ranninger, and G. De Filippis (IOS, Amsterdam, 2006).

[9] B. Gerlach and H. Löwen, Rev. Mod. Phys. 63, 63 (1991).

[10] Y. Zhao, P. Zanardi, and G. Chen, Phys. Rev. B 70, 195113 (2004); J. Sun, Y. Zhao, and W. Liang, ibid. 79, 155112 (2009).

[11] V.M. Stojanovic and M. Vanenic, Phys. Rev. B 78, 214301 (2008).

[12] V. Cataudella, G. De Filippis, and G. Iadonisi, Phys. Rev. B 60, 15163 (1999); V. Cataudella, G. De Filippis, and G. Iadonisi, ibid. 62, 1496 (2000).

[13] C.A. Perroni, V. Cataudella, and G. De Filippis, J. Phys.: Condens. Matter 16, 1593 (2004).

[14] A. Kopp and K. Le Hur, Phys. Rev. Lett. 98, 220401 (2007).

[15] See R. Ferreira, A. Berthelot, T. Grange, E. Zibik, G. Cassabois, and L. Wilson, J. Appl. Phys. 105, 122412 (2009).

[16] T. Holstein, Ann. Phys. (Leipzig) 8, 325 (1959); 8, 343 (1959).

[17] H. Fröhlich, Adv. Phys. 3, 325 (1954).

[18] A. S. Alexandrov and P. E. Kornilovitch, Phys. Rev. Lett. 82, 807 (1999).

[19] M.A. Nielsen and I.L. Chuang, Quantum Computation and Quantum Information (Cambridge University Press, Cambridge, 2000).

[20] B. Schumacher, Phys. Rev. A 51, 2738 (1995).

[21] P. Gaal, W. Kuehn, K. Reimann, M. Woerner, T. Elsaesser and R. Hey, Nature 450, 1210 (2007). 